\title{
Ionic chromophores $\mathrm{Xe}_{p}^{+}(p \leqslant 4)$ in multishell rare-gas clusters $\mathrm{Xe}_{m}\left(\mathrm{NF}_{3}\right)_{k} \mathrm{Ne}_{7500}(m, k$ $\leqslant 100$ ) studied with fluorescence spectroscopy
}

\author{
A. Kanaev ${ }^{\text {a) }}$ \\ Laboratoire d'Ingénierie des Matériaux et des Hautes Pressions, C.N.R.S., Institut Galilée, \\ Univerité Paris-Nord, Villetaneuse 93430, France \\ L. Museur \\ Laboratoire Physique des Lasers, C.N.R.S., Institut Galilée, Univerité Paris-Nord, \\ Villetaneuse 93430, France
}

\section{T. Laarmann}

Max-Born Institute, Max-Born Str. 2a, Berlin 12489, Germany

T. Möller

Institut für Atomare Physik, Technische Universität Berlin, Hardenbergstr. 36, Berlin 10623, Germany (Submitted July 7, 2006)

Fiz. Nizk. Temp. 32, 1426-1433 (November 2006)

\begin{abstract}
We have studied fluorescence spectra of small $\mathrm{Xe}_{m}$ and $\mathrm{Xe}_{m}\left(\mathrm{NF}_{3}\right)_{k}$ clusters $\left(m, k \leqslant 10^{2}\right)$ embedded in large host $\mathrm{Ne}_{7500}$ clusters, excited with SR excitation in the VUV spectral region above the $\mathrm{Xe}^{+}\left({ }^{2} P_{1 / 2}\right)$ atomic limit. The IR emission bands due to ionic chromophores $\mathrm{Xe}_{p}^{+}(p=2,3,4)$ as well as $\mathrm{UV}(B-X)$ and visible $(C-A)$ bands due to desorbed $\mathrm{Xe}^{+} \mathrm{F}^{-}$excimer molecules have been observed. The triatomic $\mathrm{Xe}_{2} \mathrm{~F}$ excimer emission has only been observed under low-energy excitation close to the lowest xenon exciton absorption band. A competition between ionic and charge-transfer reaction channels is followed as a function of the cluster sizes $m$ and $k$. (C) 2006 American Institute of Physics. [DOI: 10.1063/1.2389018]
\end{abstract}

\section{INTRODUCTION}

There is a well-known fundamental difference between neutral and ionized rare-gas clusters. The neutral clusters are bound by the weak van der Waals pairwise interaction, while polarized forces induced by the ionized core strongly increase the cluster solidity. This has a consequence on the atomic desorption process, accompanied by the cluster cooling. Another point of difference is that while in the neutral cluster the representative "building block" is the rare-gas dimer, in the ionic cluster a larger unit can be produced due to a charge delocalization process. For example, $\mathrm{Xe}_{p}^{-}$ $(p=2-4)$ units have been observed experimentally from analysis of absorption and fluorescence spectra ${ }^{1-3}$ and theoretically predicted in free xenon clusters. ${ }^{4-6}$ The IR fluorescence of self-trapped holes in rare-gas solids has been studied recently in Refs. 7-9. There has been steady interest in rare-gas ionic clusters on account of their role as model systems for understanding charge-transfer and solvation processes in bulk media. The use of clusters offers the possibility of studying the evolution from the atomic limit towards the bulk material as a function of cluster size, which is an important issue of the fundamental physics. Moreover, because of the large percentage of surface atoms, small clusters are extremely interesting objects for interface studies.

Recently, the so-called "pickup" technique has allowed the growth of small guest rare-gas clusters inside large host rare-gas clusters, and the investigation of embedded clusters with a shell-like geometric structure. ${ }^{10,11}$ This method has the advantage of controlling the temperature, the surface or bulk localization and the size of the guest cluster, as well as the size of the host cluster. Neon is a good solvent system for such experiments for several reasons: $\mathrm{Ne}_{N>1000}$ clusters are soft enough and easily pick up atoms or molecules. They efficiently thermalize the dopant molecules at a characteristic cluster temperature of $\sim 10 \mathrm{~K} .{ }^{12}$ Moreover, the surounding neon cluster atoms hardly perturb the energy levels of embedded molecules. Since neon clusters are transparent within the VUV spectral range, they are well suited to the study of electronic properties of molecules and heavier rare-gas clusters made of $\mathrm{Ar}, \mathrm{Kr}$, and $\mathrm{Xe}$ atoms. Recently, investigation of $\mathrm{Ar}_{m} \mathrm{Ne}_{N}$ (Ref. 11), $\mathrm{Kr}_{m} \mathrm{Ne}_{N}$ (Ref. 13), and $\mathrm{Xe}_{m} \mathrm{Rg}_{N}$ clusters $(\mathrm{Rg}=\mathrm{He}, \mathrm{Ne})^{14}(m \leqslant 100 \ll N \approx 7500)$ have given insight into the tightly bound bulk interface excitons in small rare-gas clusters. It has been shown that a large penetration depth of the interface exciton into the bulk of the embedded cluster, induced by the surrounding neon matrix, prohibits a formation of the electronic structure inherent to the corresponding bulk solids.

However, the solvation effect on ionic cations $\mathrm{Xe}_{p}^{-}$ $(p=2-4)$ inside multishell clusters has never been the subject of experimental study. In that way one could inspect for influence of the surrounding medium on the ionization, charge delocalization, and charge transfer processes.

In the present paper we report on the spectroscopy of small ionized clusters $(m \leqslant 100)$ embedded inside large 


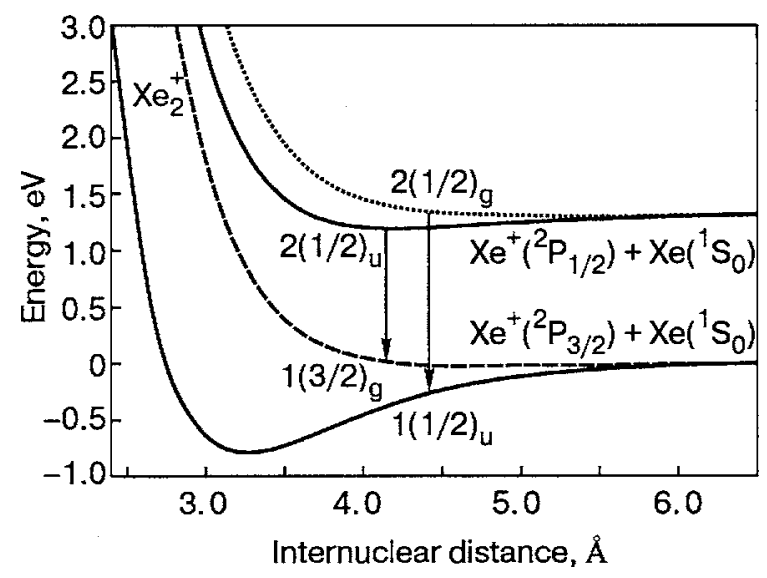

FIG. 1. Schematic potential curves of the relevant states related to $5 p^{52} P_{1 / 2}$ and $5 p^{5}{ }^{2} P_{3 / 2}$ atomic xenon ion limits.

(7500) Ne clusters. Using the multiple cross-beam technique, we have also prepared multishell $\mathrm{Xe}_{m}\left(\mathrm{NF}_{3}\right)_{k} \mathrm{Ne}_{N}$ clusters $(m, k \leqslant 100)$, where a competition between the charge delocalization onto the $\mathrm{Xe}_{p}^{-}(p=2-4)$ units and charge transfer reaction leading to the rare-gas halide excimer formation is expected. The experiments were carried out using the energy-resolved fluorescence technique. As in our previous studies, we make use of the IR fluorescence of ionic chromophores $^{2,3}$ on transitions down to the lowest bound and first excited unbound states (Fig. 1). We have followed the evolution of the IR band shape and relative IR and UV band intensities as a function of the number of picked-up Xe atoms and $\mathrm{NF}_{3}$ molecules. From a detailed analysis of the experimental data we gain new information on the charge delocalization in the solvated xenon clusters.

\section{EXPERIMENT}

The measurements have been performed at the CLULU experimental station ${ }^{15}$ at the DESY synchrotron. Neon clusters were prepared in a supersonic expansion of the gas neon at 200 mbar pressure thourgh a conical nozzle $(200 \mu \mathrm{m}$, $2 \theta=4^{\circ}$ ) cooled down to $30 \mathrm{~K}$. The average cluster size $7500 \approx N$ was determined using well-known scaling laws according to the formula: ${ }^{16,17} N=33\left(\Gamma^{*} / 1000\right)^{2.35}$, with $\Gamma^{*}$ $=K_{\mathrm{ch}} p d_{\mathrm{eq}}^{0.85} / T^{2.2875}, K_{\mathrm{ch}}(\mathrm{Ne})=185$, and $p$ in mbar, $T$ in $\mathrm{K}$, and $d$ in $\mu m$ are used. The width of the cluster size distribution (FWHM) is $\Delta N \approx N$. Using a successive "pickup" technique, these large neon clusters were doped first with $\mathrm{NF}_{3}$ molecules and second with Xe atoms from two cross jets. The embedded clusters are cold: their temperature of $10 \mathrm{~K}$ is defined by that of the neon host cluster. ${ }^{18}$ The Poisson statistic determines the average number of picked-up molecules $(k)$ and atoms $(m)$. The mean sizes of the embedded $\mathrm{Xe}_{m}$ and $\left(\mathrm{NF}_{3}\right)_{k}$ clusters have been estimated by measuring the VUVfluorescence excitation spectra of the $\mathrm{Ar}_{m}$ clusters inside large neon clusters in the range of $12.4 \mathrm{eV}$, prepared by expanding the argon gas correspondingly through xenon $(m)$ and nitrogen trifluorine $(k)$ nozzles, as is explained in Ref. 13. Laarmann et al. ${ }^{11}$ have shown that the absorption line shape of tightly bound excitons in $\mathrm{Ar}_{m}$ clusters changes with their size $m$ according to the Frenkel exciton model. By comparing the experimental spectra line shapes with those from
Ref. 11, one obtains a relation between the cross-jet pressure and the average number of embedded atoms. Since the probability for a Ne cluster to pick up atoms is dependent mainly on the Ne cluster size and the average cross-jet particle density along the beam axes, the calibration is also valid in the case of $\mathrm{Xe}$ and $\mathrm{NF}_{3}$ doping.

Monochromatized synchrotron radiation $(\Delta \lambda=0.25 \mathrm{~nm}$ bandpass) in the spectral range of 100-140 nm (Al grating) or 40-100 nm (Pt grating) was focused on a doped multishell $\mathrm{Xe}_{m} \mathrm{Ne}_{7500}$ and $\mathrm{Xe}_{m}\left(\mathrm{NF}_{3}\right)_{k} \mathrm{Ne}_{7500}$ cluster beam $10 \mathrm{~mm}$ downstream from the nozzle. Generally, we excited the cluster beam in the lowest excitonic band of the host neon clusters at $17.7 \mathrm{eV}(70 \mathrm{~nm})$. After the excitation the energy is efficiently transferred onto an impurity center, a small embedded xenon cluster. Fluorescence excitation spectra in the $\operatorname{VUV-UV}(\lambda \leqslant 300 \mathrm{~nm})$ and in the UV-visible-IR (200nm $\leqslant \lambda \leqslant 900 \mathrm{~nm}$ ) were recorded by two photomultipliers with $\mathrm{CsI}$ and $\mathrm{GaAs}(\mathrm{Cs})$ photocathodes, respectively. The background pressure was kept below $10^{-3}$ mbar during the experiments.

Before presenting experimental results, let us remark on the cluster composition. The pickup of atoms and molecules by large $\mathrm{Ne}_{7500}$ clusters results in a release of energy. Neon atoms are weakly bound and evaporate from the $\mathrm{Xe}_{m} \mathrm{Ne}_{N}$ cluster by heating. In fact, the binding energies per atom of rare-gas neon and xenon solids are $26.5 \mathrm{meV}$ and $172.3 \mathrm{meV}^{19}$ Therefore, doping may decrease the initial cluster size by $\sim 6.5 \mathrm{Ne}$ atoms per adsorbed Xe atom if the kinetic energy of the free atoms is neglected. Therefore, doping by $m \leqslant 10^{2}$ xenon atoms does not change the neon cluster size considerably. It can be shown that the same conclusion is valid for the doping by a small number of $\mathrm{NF}_{3}$ molecules. The conclusion that host neon clusters with $N=7500$ are stable under the experimental doping conditions $(m, k$ $\leqslant 100$ ) has been confirmed by measurements of the luminescence excitation spectra in the spectral range of neon absorption, $h \approx 17.7 \mathrm{eV}$.

\section{RESULTS AND DISCUSSION}

The fluorescence spectra of xenon clusters excited above the atomic ionization limit originate from molecular states related to the $5 p^{5}{ }^{2} P_{1 / 2} \rightarrow 5 p^{5}{ }^{2} P_{3 / 2}$ transitions in ionic chromophores $\mathrm{Xe}_{p}^{-}(p=2-4){ }^{2,3}$ The relevant potentials of the two-atomic ions are schematized in Fig. 1. Because of a low barrier between the trimer and tetramer ionic cores, the corresponding transitions are not resolved in relatively hot $(T$ $=79 \pm 8 \mathrm{~K})^{18}$ free xenon clusters. Experimental results on the fluorescence of $\mathrm{Xe}_{N}^{+}\left(N \leqslant 2 \times 10^{4}\right)$ have been reported in Refs. 2 and 3. However, Laarmann et al. ${ }^{3}$ have remarked that because of two main radiation channels associated with $2(1 / 2) g \rightarrow 1(1 / 2) u$ and $2(1 / 2) u \rightarrow 1(1 / 2) g$ transitions and three possible ion cores $\left(\mathrm{Xe}_{2}^{+}, \mathrm{Xe}_{3}^{+}\right.$, and $\left.\mathrm{Xe}_{4}^{+}\right)$, six fluorescence bands can be resolved in fluorescence spectra at sufficiently low temperatures. These new spectral bands have been demonstrated in small, $\mathrm{Xe}_{40}$ clusters embedded in large neon clusters using the pickup method: ${ }^{3}$ the temperature of such cluster is defined by the host neon temperature, which is about $10 \mathrm{~K} .{ }^{18}$ The size dependence of the IR fluorescence spectra of these embedded $\mathrm{Xe}_{m}$ clusters $\left(m \leqslant 10^{2}\right)$ is studied in the present work. 


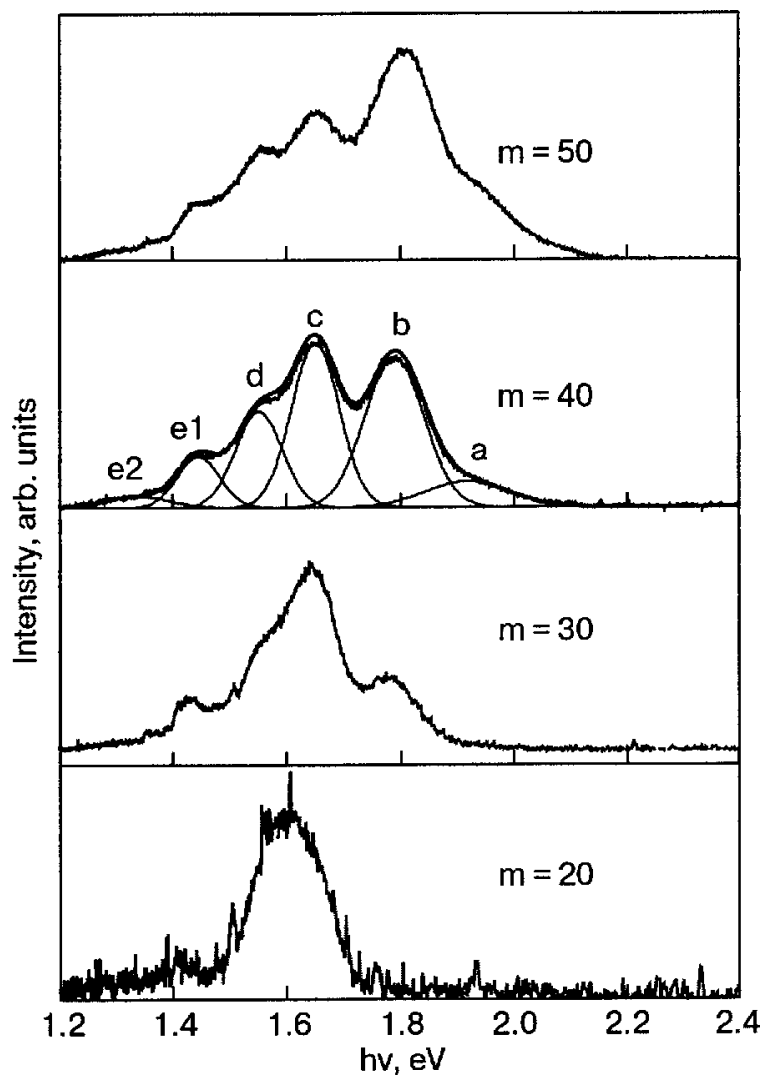

FIG. 2. Fluorescence spectra of $\mathrm{Xe}_{m} \mathrm{Ne}_{7500}$ excited at $17.7 \mathrm{eV}$.

\section{$\mathrm{Xe}_{m} \mathrm{Ne}_{7500}$ clusters}

The IR fluorescence spectra of $\mathrm{Xe}_{m} \mathrm{Ne}_{7500}$ clusters excited at $\lambda_{\text {exc }}=70 \mathrm{~nm}$ are presented in Fig. 2. The multi-peak Gaussian fit has been used to resolve the contributions of the six bands labeled by $a, b, c, d, e_{1}$, and $e_{2}$. They have been assigned by Laarmann et $a l^{3}$ to radiative transitions in the ionic dimer (subbands $\left.e_{1}, e_{2}\right)$, trimer $(b, d)$ and tetramer $(a, c)$ cores inside xenon clusters.

According to the scheme in Fig. 1, the bands $e_{1}, b$, and $a$ originate from the higher-positioned (more shallow) electronic $g$ state, whereas the bands $e_{2}, c$, and $d$ originate from the more strongly bound $u$ state: the corresponding dimer ion states are $2(1 / 2) g$ and $2(1 / 2) u$ (Ref. 20). As one can see from this figure, bands $d$ and $c$ dominate the fluorescence of very small xenon clusters, $m \leqslant 5$. Bands $b$ and $a$ appear at $m \geqslant 30$ and became stronger in clusters larger than $m \geqslant 50$. With further increase of the size, $m \geqslant 70$, the spectral shape of the IR fluorescence remains almost unchanged. Their integrated relative intensities issued from the multi-peak Gaussian fit are shown in Fig. 3. As their contribution is small, the dimer bands are not shown in this figure.

The different state origin of the two groups of bands $d / c$ and $b / a$ is evidenced from fluorescence decay measurements, presented in Fig. 4. All these curves can be well approximated by two-exponent fits, resulting in excited state lifetimes of $t_{1}=1.5 \mu \mathrm{s}$ and $t_{2}=150 \mathrm{~ns}$. On the other hand, the corresponding decay amplitudes $A_{1}$ and $A_{2}$ vary with the cluster size $m$ : only the long-lived decay component is present at $m \leqslant 20$, while the short-lived decay component intensifies in larger xenon clusters (see Fig. 4a). Its relative intensity as a function of the size $m$ is shown in Fig. 4b. A

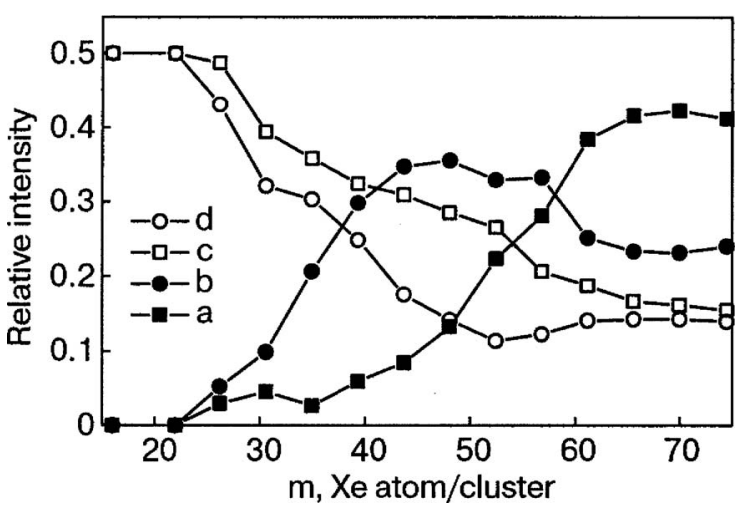

FIG. 3. Relative intensities of the IR emission bands due to ionic chromophores $(p=2,3,4)$.

correlation between relative intensity of the bands $b / a$ and short-lived fluorescence decay allows their straightforward identification. We assign the longer lifetime of $t_{1}=1.5 \mu \mathrm{s}$ to the fluorescence bands $d / c$ appearing in small xenon clusters, and the shorter lifetime of $t_{2}=150 \mathrm{~ns}$ to the fluorescence bands $b / a$ appearing in the larger clusters. This assignment is in general agreement with the dimer ion state structure dis-
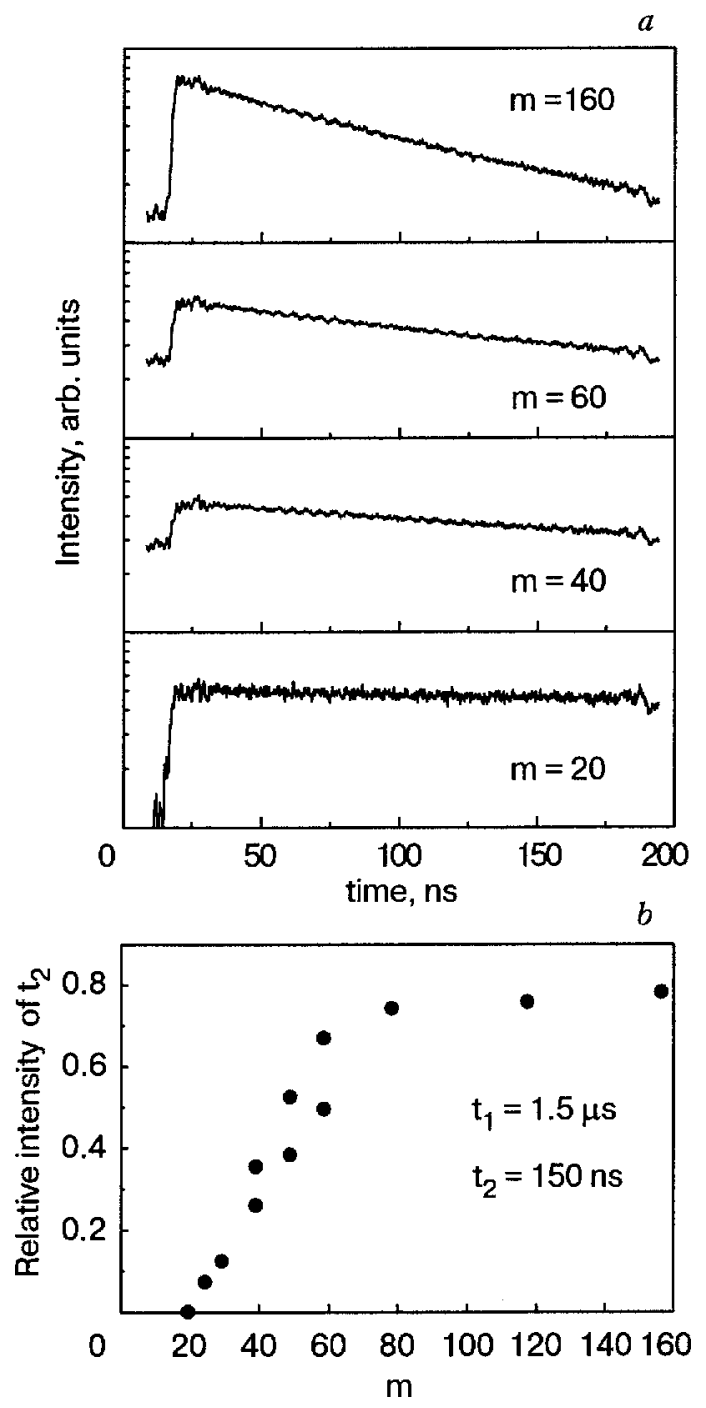

FIG. 4. IR fluorescence decay $(a)$ and the fast component relative intensity in $\mathrm{Xe}_{m} \mathrm{Ne}_{7500}$ clusters as a function of the size $m(b)\left(E_{\mathrm{exc}}=17.7 \mathrm{eV}\right)$. 
cussed in the theoretical paper by Wadt. ${ }^{21}$ Similar repartitioning of the excited electronic states is expected in xenon ion trimers and tetramers and can explain our finding.

In contrast to the ionic chromophore subbands, relative fluorescence intensity originated from different chromophores $\mathrm{Xe}_{4}^{+}$and $\mathrm{Xe}_{3}^{+}$does almost not change in the cluster size range $m \leqslant 10^{2}$. This result follows directly from Fig. 3 if we sum, respectively, the band intensities $d+b$ and $c+a$ : each chromophore accounts for about $50 \%$ of the IR intensity. Our measurements confirm the charge localization on ionic trimer and tetramer cores in small xenon clusters. Recently, Laarmann et al. ${ }^{3}$ have shown that in free $\mathrm{Xe}_{N}$ clusters the charge is preferentially repartitioned on $\mathrm{Xe}_{4}^{+}$and $\mathrm{Xe}_{3}^{+}$ cores when $N \leqslant 200$, and localized on $\mathrm{Xe}_{2}^{+}$only when $N$ $\geqslant 400$. This explains in particular why only the dimer ionic core is observed in the bulk solids in both absorption ${ }^{21}$ and fluorescence. ${ }^{22}$ Theoretical and experimental studies by Gascón $e t a l .{ }^{23}$ have shown that tetramer and trimer cores coexist in small xenon clusters $N \leqslant 30$, the relative population of which undergoes oscillatory variation with size. The interconversion of these two isomers was also suggested at temperatures of $\sim 60 \mathrm{~K}$. The temperature of our embedded $\mathrm{Xe}_{m}$ clusters is considerably lower, and the interconversion may be prohibited. This may in particular explain the fact that the subband intensity variation within each isomer with size is not accompanied by an intensity variation between the two isomers. The last is determined by the energy-relaxation dynamics and not by the thermodynamic equilibrium between the relaxed ionic cores. The isolation of $\mathrm{Xe}_{4}^{+}$and $\mathrm{Xe}_{3}^{+}$ionic isomers at the low temperature of $10 \mathrm{~K}$ makes their population independent on cluster size, in contrast to what is observed in hotter free xenon clusters $(T=79 \mathrm{~K}){ }^{3}$ We also remark that clusters with preferential isomer population cannot be resolved in our experiments because of a broad cluster size distribution: Poisson distribution of the embedded xenon clusters is convoluted with that lognormal of the host neon clusters, issued of the nozzle expansion, resulting in a width somewhat larger than $\sqrt{m}$.

Our results show the importance of the small cluster environment. Indeed, comparing with free xenon clusters $\mathrm{Xe}_{4}^{+}$ and $\mathrm{Xe}_{3}^{+}$isomers contribute in fluorescence spectra with almost equal weightings. Moreover, the upper state population within each ionic core sensitively depends on the cluster size $m$ shown in Fig. 3. This cannot be an effect of the cluster temperature, which is fixed at $10 \mathrm{~K}$. Moreover, the temperature would be expected to decrease with size $m$, leading to population of the deeper $2(1 / 2) u$ excited state. As a consequence, the intensity of the $2(1 / 2) u \rightarrow 1(1 / 2) g$ transition would increase, and that does not happen. A preferential population of the lower excited state related to $2(1 / 2) g$ of $\mathrm{Xe}_{2}^{+}$in small clusters may be explained by an effect of the interface formation similar to that discussed in Refs. 13 and 14 or by the embedded atoms compression 14. According to Refs. 13 and 14, exciton formation in embedded rare-gas clusters depends on the electron affinity. In the particular case of $\mathrm{Xe}_{m} \mathrm{Ne}_{N}$ clusters a perturbation of the Xe-Ne interfacial layer is strong, and the bulk exciton of $\mathrm{Xe}_{m}$ only appears at sufficiently large $m \geqslant 50$. The second case seems less probable, since an effect of compression was observed only in small neon clusters, about $40<N<90 .{ }^{14}$ However, the

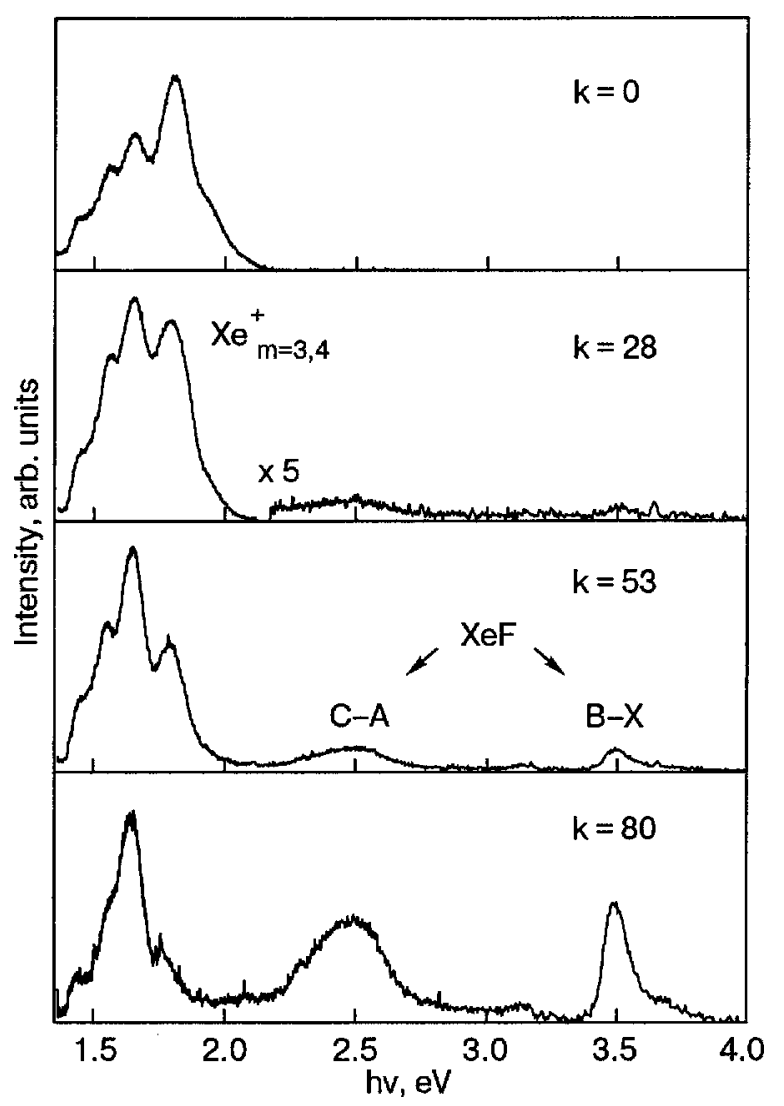

FIG. 5. Fluorescence spectra after excitation of multishell $\mathrm{Xe} 50\left(\mathrm{NF}_{3}\right)_{k} \mathrm{Ne}_{7500}$ clusters at $17.7 \mathrm{eV}$.

case of embedded ionized cluster atoms is different from that of neutral isolated xenon atoms studied in Ref. 14, and further studies are requiresd.

\section{$\mathrm{Xe}_{m}\left(\mathrm{NF}_{3}\right)_{k} \mathrm{Ne}_{7500}$ clusters}

A competition between the ionization and chargetransfer reaction can be studied in multishell $\mathrm{Xe}_{m}\left(\mathrm{NF}_{3}\right)_{k} \mathrm{Ne}_{7500}$ clusters. In fact, excitation of the neon cluster activates two reaction mechanisms. (1) Ionization of the internal $\mathrm{Xe}_{m}$ cluster takes place following the energy transfer process. The electron leaves the cluster, whereas the hole is localized on excited chromophores $\mathrm{Xe}_{p}^{+^{*}}(p=2,3,4)$ that give rise to the characteristic IR fluorescence bands shown in Fig. 2. (2) The energy transferred from neon cluster triggers charge-transfer reactions resulting in excimer creation: $\mathrm{XeF}$ or $\mathrm{Xe}_{2} \mathrm{~F}$. In bulk xenon $\mathrm{XeF}$ relaxes to $\mathrm{Xe}_{2} \mathrm{~F}$, and therefore its observation would be an indication of the excited-state desorption process. ${ }^{24,25}$

The fluorescence spectra recorded after excitation of multishell $\mathrm{Xe}_{50}\left(\mathrm{NF}_{3}\right)_{k} \mathrm{Ne}_{7500}$ clusters at $17.7 \mathrm{eV}$ are shown in Fig. 5 for different sizes $k$. At $k=0$ only IR fluorescence of $\mathrm{Xe}_{p}^{+*}$ has been observed. However, with an increase of $\mathrm{NF}_{3}$ doping the IR band shape changes. It seems to follow the opposite tendency to that earlier observed in the twocomponent $\mathrm{Xe}_{m} \mathrm{Ne}_{7500}$ clusters: with increasing $k$ the intensity of the subbands $a$ and $b$ decreases relative to that of subbands $c$ and $d$. Moreover, at $k \leqslant 30$ new $\mathrm{XeF}$ excimer emission bands appear: $B-X$ in the $\mathrm{UV}$ at $3.5 \mathrm{eV}$, and $C-A$ in the visible at $2.5 \mathrm{eV}$. 
The observed excimer bands apparently belong to the desorbed excited fragments illustrating the importance of the excited-state stimulated desorption processes in cryogenic solids. The relatively weak spectral features at $3.65 \mathrm{eV}$ and $3.15 \mathrm{eV}$ do not correspond to the known $\mathrm{NF}_{3}$ fluorescence products. ${ }^{26}$ They indicate that a part of the expulsed excited products is hot and possess significant vibrational excitation: corespondingly, $v^{\prime}=2-3$ ( $B$ state $)$ and $v^{\prime}=6 \pm 1$ ( $C$ state). The XeF excimers escape through the outer shell of $\mathrm{Ne}_{7500}$ cluster. However, according to Ref. 12 vibrational cooling $\mathrm{OF}$ fragments can be particularly inefficient in neon clusters in view of the low Debye energy $(6.4 \mathrm{meV})$ compared to the vibrational quantum (e.g., in the $C$ state $\omega_{e}=346 \mathrm{~cm}^{-1}$ ). The main fluorescent bands however are narrower than those observed earlier in pure xenon clusters. ${ }^{24,25}$ This may be an effect of rotational cooling by the neon matrix.

The mechanism of the excimer state creation (channel (2)) may proceeds as follows:

$$
\begin{aligned}
\mathrm{Ne}_{N}^{*} \rightarrow & \left(\mathrm{Xe}_{m}^{* *}+e^{-}\right) \stackrel{(1)}{\longrightarrow} \mathrm{Xe}_{p}^{+*}+e_{\text {free }}^{-} \stackrel{(2)}{\longrightarrow} \mathrm{Xe}_{p}^{+*} \\
& +\left(\mathrm{NF}_{3}\right)_{k}^{-} \rightarrow \mathrm{XeF}(B, C)_{\text {free }}+\text { products }
\end{aligned}
$$

First, the ionization of the internal $\mathrm{Xe}_{m}$ cluster takes place. In $\mathrm{Xe}_{m} \mathrm{Ne}_{N}$ clusters this results in $\mathrm{Xe}_{p}^{+*}$ center creation (channel (1)). However in $\mathrm{Xe}_{m}\left(\mathrm{NF}_{3}\right)_{k} \mathrm{Ne}_{N}$ the electron may not escape and can be trapped by the first-shell cluster $\left(\mathrm{NF}_{3}\right)_{k}$. This happens if the size $k$ is above some critical value $k^{*} \approx 30$. The $\mathrm{NF}_{3}$ molecule accommodating excess electron subjects to the dissociative attachment. However, it can be stabilized in clusters as $\mathrm{NF}_{3}^{-}$anion and larger cluster ion units $\left(\mathrm{NF}_{3}\right)_{l>1}^{-}$ (Ref. 24). In such a case the Coulomb attraction between the localized hole $\mathrm{Xe}_{p}^{+*}$ and $\left(\mathrm{NF}_{3}\right)_{k}^{-}$or lighter anionic fragment can result in $\mathrm{XeF}$ excimer formation. The excess energy of the electronically excited center is apparently so strong that the expulsion proceeds before the relaxation into $\mathrm{Xe}_{2} \mathrm{~F}$ takes place. Additionally, the binding energy of the $\mathrm{Xe}_{p}^{+*}$ core is much smaller than that of the ground-state ion $\mathrm{Xe}_{p}^{+}$. This may prohibit $\mathrm{Xe}_{2} \mathrm{~F}$ formation from xenon core states related to the $5 p^{5} P_{1 / 2}$ atomic limit. In fact, no signature of the of this trimer rare-gas halide emission has been observed with $17.7 \mathrm{eV}$ excitation.

On the other hand, if the excitation energy is lowered below the onset of ionization, the broadband intracluster emission of $\mathrm{Xe}_{2} \mathrm{~F}$ appears, as shown in Fig. 6. It is centered at $1.48 \mathrm{eV}(838 \mathrm{~nm})$, which is lower than that reported in bulk solid Xe matrixes, $1.60 \mathrm{eV}(775 \mathrm{~nm}),{ }^{25}$ and in small free xenon clusters, $1.54 \mathrm{eV}(805 \mathrm{~nm}) .^{22}$ The spectral width of this emission $\Delta E_{F W H M}=0.27 \mathrm{eV}$ corresponds to that observed in solids and somewhat smaller that in free clusters $(0.35 \mathrm{eV})$. A small red shift in the embedded xenon clusters and a narrower bandwidth may be due to the neon cluster compression. This issue requires verification.

The dependence of the $\mathrm{Xe}_{p}^{+*}$ fluorescence intensity $\left(I_{I R}\right)$ on the size $k$ of the first-shell cluster $\left(\mathrm{NF}_{3}\right)_{k}$ is shown in Fig. 7. In this series the integral IR fluorescence of the excited chromophores $(p=2,3,4)$ was recorded through the RG610 filter (red cut-off at $\lambda>600 \mathrm{~nm}$ ), which allowed highersensitivity measurements in a large size domain. The observed semilogarithmic plot is characterized by two kinetics domains separated by a crossover region at $30 \leqslant k<60$. This

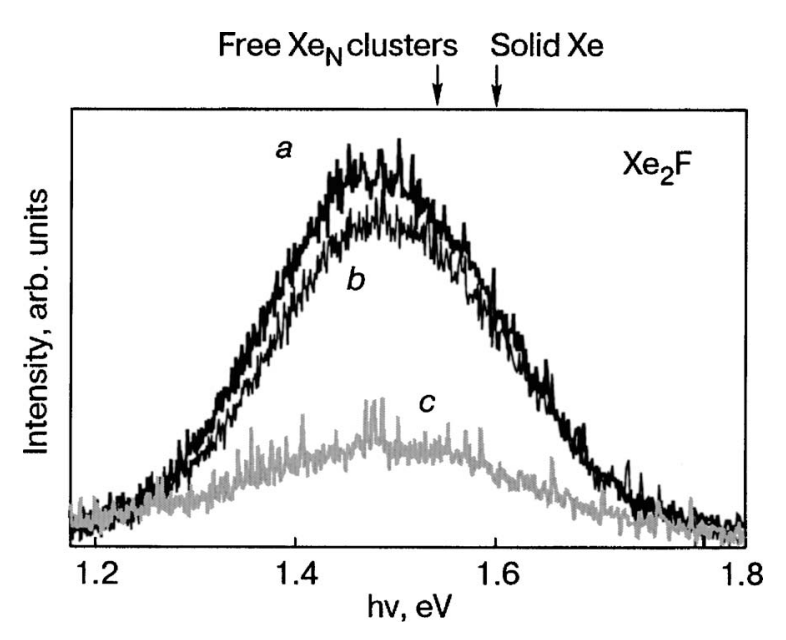

FIG. 6. IR fluorescence spectra after excitation of multishell $\mathrm{Xe} 50\left(\mathrm{NF}_{3}\right)_{k} \mathrm{Ne}_{7500}$ clusters at $8.551 \mathrm{eV}$ for $k=50(a), 25(b), 14(c)$.

transient region apparently relates to an increase of the electron trapping rate by the $\mathrm{NF}_{3}$ cluster after the critical size of $k \geqslant k^{*}=30$. The fact that the critical size $k^{*}$ does not depend appreciably on the size of the embedded xenon cluster $m$ (see Fig. 7) indicates that the electron exit from the neon cluster may not be a direct but rather a delayed process: in case of the direct exit the electron trapping probability would be a function of the $\mathrm{NF}_{3}$ shell width $\left(k^{2 / 3}\right)$ and not of the size $k$. An increase of the neutral reaction channel relative to molecular ionization has recently been observed in large neon clusters doped with water molecules. ${ }^{12}$

Let us make an additional remark concerning the excited-state reactivity of $\mathrm{Xe} p^{+^{*}}$. The plot of $\ln \left(I_{I R}\right)$ versus cluster size $k$ suggests an excited-states quenching process. Moreover, as we can see in Fig. 5, the spectral line shape of the IR band changes with growing $k$ : subbands $b$ and $a$ weaken faster, and the subbands $d$ and $c$ dominate the fluorescence spectra at large $k$. As our results show, the shortlived excited state $2(1 / 2) g$ is at the origin of the subbands $b$ and $a$, while the long-lived excited state $2(1 / 2) u$ is at the origin of the subbands $d$ and $c$. This indicates a much higher reactivity of the $2(1 / 2) g$ state in comparison to the $2(1 / 2) u$ one. This may be related to its larger equilibrium distance, which enables more efficient potential curve-crossing be-

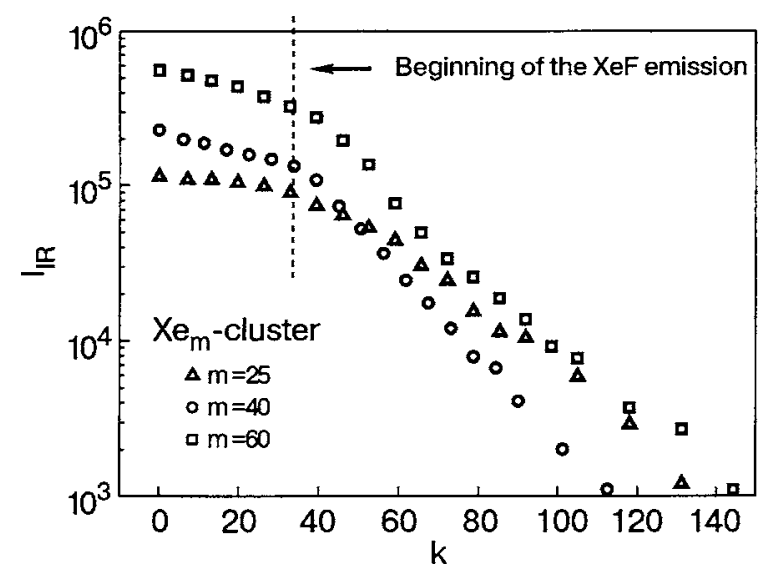

FIG. 7. Relative intensity of the IR fluorescence of $\mathrm{Xe}_{m}\left(\mathrm{NF}_{3}\right)_{k} \mathrm{Ne}_{7500}$ clusters as a function of the size $k, E_{\mathrm{exc}}=17.7 \mathrm{eV}$. 
tween the chromophore and the anion. Indeed, the more localized state $2(1 / 2) u$ may be centered inside the embedded clusters while the more delocalized ${ }^{2}$ one $2(1 / 2) g$ is readily set in contact with the negatively charged halogen donor.

\section{CONCLUSION}

The present spectroscopic study of multishell $\mathrm{Xe}_{m}$ and $\mathrm{Xe}_{m}\left(\mathrm{NF}_{3}\right)_{k}$ clusters $\left(m, k \leqslant 10^{2}\right)$ embedded in large host $\mathrm{Ne}_{7500}$ clusters clears up the hole localization process in small xenon clusters and evidences a competition between the ionization and charge-transfer reactions. The IR emission bands due to ionic chromophores $(p=2,3,4)$ as well as the $\mathrm{UV}(B-X)$ and visible $(C-A)$ bands due to desorbed $\mathrm{Xe}^{+} \mathrm{F}^{-}$ excimer molecules have been observed. The cluster size effect of $m$ and $k$ on the reaction dynamics was observed. The delocalization of hole on trimer and tetramer ionic cores is confirmed in xenon clusters of size $m \leqslant 10^{2}$. Because of a low cluster temperature of $10 \mathrm{~K}$, configuration mixing is prohibited, resulting in almost equal relative ion isomer populations. However, the excited state distribution within each isomer changes with the size $m$, which may reflect the compression of the atoms or an Xe-Ne interface effect. The free $\mathrm{XeF}$ excimer emission shows the importance of the excited-state desorption processes in rare-gas clusters. The charge-transfer reaction takes place when the first-shell cluster size $k$ is higher than the critical size $k^{*} \approx 30$. The triatomic $\mathrm{Xe}_{2} \mathrm{~F}$ excimer is not observed at $17.7 \mathrm{eV}$ excitation; this emission has only been observed under low-energy excitation close to the lowest atomic ${ }^{3} P_{1,2}$ states. This result shows that a large neon cluster is not a good cage for rapid reaction fragments, which readily escape it.

This work was supported by the IHP-Contract HPRI-CT1999-00040 of the European Commission.

${ }^{a}$ E-mail: kanaev@limhp.univ-paris13.fr

${ }^{1}$ H. Haberland, B. von Issendorff, T. Kolar, H. Kornmeier, C. Ludewigt, and A. Risch, Phys. Rev. Lett. 67, 3290 (1991).

${ }^{2}$ A. V. Kanaev, M. C. Castex, L. Museur, R. von Pietrowski, and T. Möller, Phys. Rev. Lett. 75, 2674 (1995).

${ }^{3}$ T. Laarmann, A. Kanaev, K. von Haeften, H. Wabnitz, R. von Pietrowski, and T. Möller, J. Chem. Phys. 116, 7558 (2002).

${ }^{4}$ N. L. Doltsinis, Mol. Phys. 97, 847 (1999).

${ }^{5}$ J. A. Gascon, R. W. Hall, C. Ludewigt, and H. Haberland, J. Chem. Phys. 117, 8391 (2002).

${ }^{6}$ D. Hrivnak and R. Kalus, Chem. Phys. 278, 21 (2002).

${ }^{7}$ E. V. Savchenko, N. Gaspary, A. Lammers, and V. E. Bondybey, J. Low Temp. Phys. 111, 693 (1998).

${ }^{8}$ E. V. Savchenko, O. N. Grigorashchenko, O. M. Sokolov, J. Agreiter, N. Gaspary, A. Lammers, and V. E. Bondybey, J. Electron. Spectr. 101-103, 377 (1999).

${ }^{9}$ E. V. Savchenko, A. N. Ogurtsov, O. N. Grigorashchenko, A. Beyer, M. Lorenz, A. Lammers, and V. E. Bondybey, Nucl. Instrum. Methods Phys. Res. B 166-167, 47 (2000).

${ }^{10}$ T. Laarmann, K. von Haeften, H. Wabnitz, and T. Möller, J. Chem. Phys. 118, 3043 (2003).

${ }^{11}$ T. Laarmann, K. von Haeften, A. Kanaev, H. Wabnitz, and T. Möller, Phys. Rev. B 66, 205407 (2002).

${ }^{12}$ A. Kanaev, L. Museur, F. Edery, T. Laarmann, and T. Möller, Eur. Phys. J. D 20, 261 (2002).

${ }^{13}$ A. Kanaev, L. Museur, F. Edery, T. Laarmann, and T. Möller, Phys. Rev. B 69, 125343 (2004).

${ }^{14}$ R. von Pietrowski, K. von Haeften, T. Laarmann, T. Möller, L. Museur, and A. Kanaev, Eur. Phys. J. D 38, 323 (2006).

${ }^{15}$ R. Karnbach, M. Joppien, J. Stapelfeldt, J. Wormer, and T. Möller, Rev. Sci. Instrum. 64, 2838 (1993).

${ }^{16}$ O. F. Hagena, Z. Phys. D: At., Mol. Clusters 4, 291 (1987).

${ }^{17}$ U. Buck and R. Krohne, J. Chem. Phys. 105, 5408 (1996).

${ }^{18}$ J. Farges, M. F. De Feraudy, B. Raoult, and G. Torchet, Surf. Sci. 106, 95 (1981).

${ }^{19}$ G. Zimmerer, in: Excited-State Spectroscopy of Solids, U. M. Grassano and N. Terzi (eds.), Amsterdam, N. Holland (1987), p. 37.

${ }^{20}$ Y. Lu, Y. Morioka, T. Matsui, T. Tanaka, H. Yoshii, R. I. Hall, T. Hayaishi, and K. Ito, J. Chem. Phys. 102, 1553 (1995).

${ }^{21}$ W. R. Wadt, J. Chem. Phys. 68, 402 (1978); W. R. Wadt, J. Chem. Phys. 73, 3915 (1980).

${ }^{22}$ E. V. Savchenko, O. N. Grigorashchenko, O. M. Sokolov, J. Agreiter, N. Caspary, A. Lammers, and V. E. Bondybey, J. Electron Spectrosc. Relat. Phenom. 101-103, 377 (1999).

${ }^{23}$ J. A. Gascon, R. W. Hall, C. Ludewigt, and H. Haberland, J. Chem. Phys. 117, 8391 (2002).

${ }^{24}$ R. von Pietrowsky, M. Lengen, L. Moussavizadeh, L. Museur, A. V. Kanaev, M. C. Castex, and T. Möller, Faraday Discuss. 108, 175 (1997).

${ }^{25}$ L. Moussvizadeh, K. von Haeften, L. Museur, A. V. Kanaev, M. C. Castex, R. von Petrovsky, and T. Möller, Chem. Phys. Lett. 305, 327 (1999).

${ }^{26}$ D. P. Seccombe, R. P. Tuckett, H.-W. Jochims, and H. Baumgartel, Chem. Phys. Lett. 339, 405 (2001).

${ }^{27}$ N. Ruckhaberle, L. Lehmann, S. Matejcik, E. Illenberger, Y. Bouteiller, V. Periquet, L. Museur, C. Desfrancois, and J.-P. Schermann, J. Phys. Chem. A 101, 9942 (1997).

${ }^{28}$ M. E. Fajardo and V. A. Apkarian, J. Chem. Phys. 85, 5660 (1986).

This article was published in English in the original Russian journal. Reproduced here with stylistic changes by AIP. 